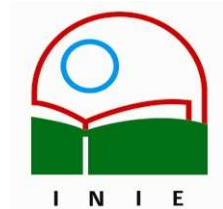

Actualidades Investigativas en Educación

Revista Electrónica publicada por el

Instituto de Investigación en Educación

Universidad de Costa Rica

ISSN 1409-4703

http://revista.inie.ucr.ac.cr

COSTA RICA

\title{
LA SIMULACIÓN COMPUTARIZADA COMO INSTRUMENTO DEL MÉTODO EN EL PROCESO DE ENSEÑANZA Y APRENDIZAJE DE LA FÍSICA, DESDE LA COGNICIÓN SITUADA: LEY DE OHM
}

COMPUTERIZED SIMULATION, AS AN INSTRUMENT USED AS THE METHOD IN THE EDUCATION PROCESS AND IN LEARNING PHYSICS, FROM SITUATED COGNITION:

LAW OF OHM

Volumen 8, Número 1

pp. 1-31

Este número se publicó el 30 de abril 2008

Germán Amaya Franky

La revista está indexada en los directorios:

LATINDEX, REDALYC, IRESIE, CLASE, DIALNET, DOAJ, E-REVIST@S,

La revista está incluida en los sitios:

REDIE, RINACE, OEI, MAESTROTECA, HUASCARAN

Los contenidos de este artículo están bajo una licencia Creative Commons

(c) $\stackrel{(1)(9)}{\mathrm{EY}}$ 


\title{
LA SIMULACIÓN COMPUTARIZADA COMO INSTRUMENTO DEL MÉTODO EN EL PROCESO DE ENSEÑANZA Y APRENDIZAJE DE LA FÍSICA, DESDE LA COGNICIÓN SITUADA: LEY DE OHM COMPUTERIZED SIMULATION, AS AN INSTRUMENT USED AS THE METHOD IN THE EDUCATION PROCESS AND IN LEARNING PHYSICS, FROM SITUATED COGNITION: LAW OF OHM
}

Resumen: Este escrito presenta a modo de resumen, los resultados de una investigación que se desarrolló al marco de las actividades metodológicas dentro del proceso de enseñanza y aprendizaje de las ciencias, donde las nuevas tecnologías son las protagonistas.

Se trata de establecer un análisis teórico, apoyado por datos, en el que se determinen las potencialidades de los entornos de simulación como espacios que pueden ayudar a generar soluciones y alternativas al problema de la descontextualización del aprendizaje de la física. Esto, como una problemática presente en una gran parte de las actividades educativas desarrolladas en contextos institucionales.

Palabras clave: DESCONTEXTUALIZACIÓN, SIMULADORES COMPUTARIZADOS, ACTIVIDAD, SITUACIÓN, CONTEXTO, COGNICIÓN SITUADA.

\begin{abstract}
This document is a summarization of results from an investigation developed within methodological activities of teaching and learning in the fields of science, where new technology is the protagonist.

It analyzes from a theoretical point of view, supported by data that shows the potential of simulated surroundings as tools that can help generate solutions and be alternatives to the problems of de-contextualization learning in physics. This problem of de-contextualization is present in a majority of educational activities developed in institutional contexts.
\end{abstract}

Key words: DE-CONTEXTUALIZATION, COMPUTERIZED SIMULATORS, ACTIVITY, SITUATION, CONTEXT, AND SITUATED LEARNING.

\section{Introducción.}

Es absolutamente posible adquirir una herramienta pero no poder utilizarla. De la misma manera, es común que los estudiantes adquieran los algoritmos, rutinas y descontextualicen las definiciones que no pueden utilizar y que, por lo tanto, se tornan inertes. Desafortunadamente, este problema no es siempre evidente, por ejemplo: los estudiantes en una clase de circuitos eléctricos, podrán aprender mucho sobre intensidad, tensión, resistencia y conocer la relación establecida por Ohm en un circuito eléctrico, pero a la vez, pueden ignorar completamente cómo podrían usar este conocimiento en una situación real.

\footnotetext{
Licenciado en Pedagogía, Universidad de Pamplona, Colombia; Especialista en Informática educativa de la Universidad Francisco de Paula Santander, Colombia; Doctor en procesos de Formación en Espacios Virtuales de la Universidad de Salamanca, España. Profesor de tiempo completo adscrito a la Facultad de Educación en la Universidad de Pamplona, Colombia y miembro del Grupo de Investigación Pedagógica.
}

Dirección electrónica: german.amaya@unipamplona.edu.co

Artículo recibido: 25 de febrero, 2008

Aprobado: 21 de abril, 2008 
Gran parte de esta carencia significativa se debe a la descontextualización que genera todo aprendizaje institucionalizado. Cabe resaltar que las actividades descontextualizadas son todas aquellas que se desarrollan fuera del contexto de uso y aplicación social, es decir, un proceso que se ejecuta lejos de situaciones reales de la vida cotidiana en la que se aprehenden los significados.

La mayoría de aportes teóricos que se especializan en indagar la manera como los humanos construyen el conocimiento, resaltan la necesidad de desarrollar actividades en contextos concretos que permitan a los individuos encontrarle significado a lo que se aprende. En tal sentido, los eventos reales aportan un entorno de posibilidades situacionales, de significado sociocultural y de actividad específica, que facultan la construcción de un aprendizaje significativo.

La educación tradicional ha buscado por siempre adoptar marcos teóricos y metodologías de enseñanza y aprendizaje que permitan hacer de las aulas de clase un entorno significativo para las actividades pedagógicas ${ }^{2}$. Para ello, ha construido las actividades mediante aproximaciones directas a acontecimientos y situaciones del mundo real y programaciones organizadas mediante el empleo de las posibilidades estructuradoras de los textos escritos. La construcción de estas actividades en vista al aprendizaje significativo, constituye un verdadero reto pedagógico, pues la difícil tarea de generar eventos realistas y significativos en el interior de las aulas de clase, termina siendo un objetivo y una fuente de dificultad para el proyecto de la comprensión significativa del mundo dentro de contextos institucionalizados de aprendizaje.

Como proyecto pedagógico en contextos de clausura institucional, surgen

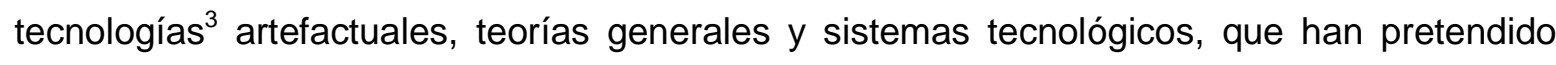
dar respuesta a dicha problemática. Elementos tales como las videocintas, proyectores, maquetas y modelos de realidad, entre otros, han sido instrumentos utilizados durante décadas; sin embargo, se trata de contextos que, en mayor o menor medida, sólo parcialmente consiguen superar la descontextualización que origina todo aprendizaje formal

2 En palabras de Wenger: "la práctica es el proceso por el cual podemos experimentar el mundo y nuestro compromiso con él como algo significativo" (Wenger, 2001, p. 75). 
institucionalizado (Agnew, \& Glen, 1990; Kinzie, Strauss \& Foss, 1993; Jude, 1999; Michelsen, 2004).

Al respecto, en esta época y gracias a los adelantos en materia de nuevas tecnologías de la información y la comunicación (NTIC), florecen grandes y positivas expectativas, porque los entornos virtuales de aprendizaje permiten simular eventos reales que pueden ser un aporte metodológico a las descontextualizadas actividades educativas dentro de la educación institucionalizada.

Estos entornos de simulación, que se hallan inscritos bajo el nombre de simuladores computarizados, pueden ser una alternativa metodológica de solución a la descontextualización del aprendizaje: Cabe resaltar que, por simulación se entiende "toda la teoría relacionada con el proceso en el cual se sustituyen las situaciones reales por otras creadas artificialmente", pudiendo representar en el modelo la apariencia, la estructura, la organización de sus componentes y la dinámica del sistema. (González, 1990, p. 284). La simulación computarizada es la representación digital de un sistema real, expresada mediante otro sistema manipulable que puede ser natural, artificial o imaginario.

De la misma forma, en el plano teórico emergen tecnologías del pensamiento que resaltan el contexto situacional y el proceso dinámico de la actividad como ejes en la construcción del conocimiento. Un ejemplo de estos marcos teóricos es la cognición situada. Este marco de inteligibilidad resalta el hecho que "el conocimiento está situado, siendo en parte un producto de actividad, del contexto y de la cultura en la cual se desarrolla y se utiliza" (Brown, Collins \& Duguid, 1989, pp. 33-42). La cognición situada demanda que las actividades deben ser sugeridas por un contexto de realidad situacional, sociocultural y de actividad, para que las interacciones y demás elementos del contexto, permitan a los educandos encontrar significado a lo que se aprende. Desde esta perspectiva, se resalta el carácter fundamental de los entornos reales en la construcción del conocimiento.

\footnotetext{
${ }^{3}$ Sancho define la tecnología como "el conjunto de saberes que nos permite intervenir en el mundo, como el conjunto de herramientas físicas o artefactuales, psíquicas o simbólicas y sociales u organizativas" (Sancho, 1994, p.7). 
Estos hechos exhortan a buscar alternativas de solución que permitan generar eventos realistas y contextos de actividad situada en espacios como las aulas de clase o entornos digitales de educación institucionalizada.

Este trabajo pretende identificar el posible potencial de la simulación computarizada de la realidad, vista como instrumento del método en la construcción significativa del conocimiento; es decir, espacios virtuales de simulación que pueden ser la solución a la descontextualización del aprendizaje que se genera al marco de la educación institucionalizada.

Desde lo anterior, se hace necesario abordar dos grandes espacios en este trabajo: el primero tiene relación con la interpretación pedagógica de los entornos de simulación como instrumentos del método y espacios que posibilitan la contextualización del aprendizaje. Como segundo, se establece un estudio empírico que permita recolectar datos, con el fin de establecer las posibilidades de los softwares de simulación para generar contextos significativos de enseñanza y aprendizaje.

\section{Referente teórico}

\subsection{La cognición situada, marco de inteligibilidad tecnológico}

La necesidad de establecer conexiones directas entre contexto y sujeto, como elemento esencial para la construcción consciente del conocimiento, es una idea que tiene sus raíces en la teoría sociocultural (Vygotsky, 1979). Sin embargo, fue en 1989 cuando el concepto de aprendizaje situado toma forma y nombre, gracias a un escrito presentado por Brown, J., Collins, A. \& Duguid, P. (1989), quienes después de anunciar el abandono del paradigma del procesamiento de información, deciden sustituirlo por otro denominado Aprendizaje Situado.

Brown, J. y sus colaboradores, desaprueban las actividades educativas y la adquisición de conceptos lejos de los ambientes en los cuales el aprendizaje tiene pertenencia; es decir, lejos de donde se aprende y se utiliza. Los autores manifiestan que "el conocimiento está situado, siendo en parte un producto de la actividad, del contexto y de la cultura en la cual se desarrolla y se utiliza" (Brown, 1989, pp. 33-42). Estos autores defienden la premisa que sustenta que en el aprendizaje se deben tener presentes todos los elementos situacionales propios de la actividad en la cual se desarrolla y se aplica el 
conocimiento, porque esos elementos son inseparables de las situaciones. Con ello, afirman que el aprendizaje es un producto de la actividad, "porque las actividades son integrales a la cognición y al aprender" (Brown, 1989, pp. 33-42).

Los estudiantes pueden aprobar los exámenes (una parte distintiva de la cultura de la escuela) pero sin poder aún utilizar las herramientas conceptuales de un dominio en prácticas auténticas (...), se debe destacar que para aprender estos temas y no aprender sobre ellos, los estudiantes necesitan conceptos mucho más que abstractos y ejemplos autónomos. Necesitan ser expuestos al uso de las herramientas conceptuales de un dominio en actividad auténtica (Brown, 1989, pp. 33-42).

El aprendizaje situado es visto como una actividad natural y cotidiana del conocimiento humano. Este tipo de aprendizaje se caracteriza porque la construcción del conocimiento se genera de forma dinámica, mediante la interacción con la situación. La cognición situada es el concepto de la actividad dentro de una matriz de formas sociales que motivan los pensamientos, actos, acciones y decisiones; es decir, la acción se sitúa en el papel de los miembros de una comunidad. (Clancey, 1995; Lave \& Wenger, 1992).

La cognición situada se especializa en indagar cómo el conocimiento se adquiere en dependencia de un contexto situacional y cómo influyen los elementos de éste en la construcción de un aprendizaje significativo ${ }^{4}$. La participación, la interacción y el compromiso del aprendiz, son características esenciales en la construcción consciente del conocimiento. En la cognición situada el aprendizaje se torna situado por la especificidad generada de los acontecimientos irrepetibles y la interpretación cultural que se generan dentro de las situaciones. Por tanto, el aprendizaje está situado en dependencia del contexto situacional, de la actividad, y del contexto socio-cultural que los individuos comparten en el momento que se construye dicho aprendizaje; que a su vez, es herramienta que permite el desarrollo de la actividad. 


\subsection{La simulación computarizada}

La simulación se define como un "acontecimiento estructurado que involucra relaciones causales entre el elemento y evento que representa una situación del mundo real" (Duchastel 1990, en Martí, 1992). La simulación es la reproducción de una situación o un fenómeno que se presenta generalmente simplificado y, que a su vez, permite la manipulación de sus variables intervinientes. En tal sentido, las simulaciones deben constituir un "modelo de situación o de fenómeno, en el que aparecen los aspectos que se consideran importantes para nuestro propósito, despreciando así los que son secundarios o accesorios" (Delval, 1986, p. 154). Por tanto, un entorno o modelo de simulación pretende representar el desarrollo de la realidad en forma parcial, procura representar en parte el funcionamiento de un sistema real.

\subsubsection{Potencialidades pedagógicas de los entornos de simulación}

Los antecedentes permiten entrever que los entornos de simulación son recursos que presentan diversos aportes al sector de la educación. Sin embargo, la efectividad de las herramientas utilizadas en el proceso de enseñanza y aprendizaje no depende sólo de los artefactos, sino que existen muchos otros factores que influyen directamente, tales como, estilos, ritmos, preferencias, capacidades, estrategias de aprendizaje y antecedentes historiales del aprendiz con relación a los conocimientos previos. Por consiguiente, "el aprendizaje depende esencialmente del ser que aprende, no de forma directa de lo que desea o se propone el que enseña" (Gimeno, y Pérez, 1992). Desde esta posición, se hace necesario reconocer en los contextos un elemento potenciador de las motivaciones que conllevan a generar aprendizaje significativo.

El entorno educativo adecuadamente prediseñado, potencia las posibilidades de los individuos para la construcción del conocimiento, porque el factor pedagógico de los entornos depende en gran medida de la metodología empleada; entendida ésta como "la ciencia que estudia los métodos, técnicas, procedimientos y medios dirigidos a la enseñanza de una disciplina dada". Así, las tecnologías deben ser vistas como herramientas para instrumentar los métodos y no como métodos propiamente dichos (Arias, 2004).

\footnotetext{
4 "La significatividad de un aprendizaje se alimenta de las vivencias del aprendiz, tanto en el aspecto cognitivo como en el psicológico, social y cultural” (Soler, E. 2002, pp. 1-22).
} 
El uso de elementos tecnológicos, para este caso, los simuladores computarizados, debe ser visto como un recurso que media y faculta a los aprendices en la construcción del conocimiento. Las simulaciones computarizadas deben ser vistas como recursos técnicos de orden material, que le facilitan al educando la interacción, estudio, y/o modelación de la realidad o de una parte de ésta. Desde esta perspectiva, los ordenadores personales constituyen medios técnicos de especial significación para el contexto metodológico actual y no elementos pedagógicos por sí solos. En definitiva, el valor pedagógico y didáctico de los entornos de simulación, es proporcionado por el contexto metodológico en el que se explotan sus cualidades.

Ahora bien, si la simulación es vista como un recurso que facilita la ejecución de metodologías educativas y, por consiguiente, de procedimientos de aprendizaje ¿por qué no se deben clasificar como metodología propiamente dicha?

Para que la simulación se pueda clasificar como una metodología, tendría que ser por sí sola un elemento suficiente para penetrar en la esencia de la realidad, y esto no es así, pues la modelación de un evento real no es un hecho suficiente para que se produzcan actividades de interacción significativa. Desde aquí, se hace necesario el empleo de procedimientos que en forma secuencial ayuden a la interpretación de esa realidad. Por otra parte, y desde un enfoque sociocultural del aprendizaje, las simulaciones por sí solas no pueden generar eventos e interacciones sociales o sistemas colaborativos; es cierto que sí generan un entorno común de significados, pero en dependencia de un proceso metodológico que induce a la reflexión. En consecuencia, se deben reconocer las simulaciones como elementos que coaccionan con la metodología en el proceso de enseñanza y aprendizaje, porque las simulaciones son instrumentos del método que corresponden a una parte de las actividades que comprenden un diseño metodológico y, no son por sí solas metodología de enseñanza y aprendizaje.

\subsubsection{Características pedagógicas de los entornos de simulación: una visión desde} la cognición situada

En este aparte se analizan los entornos de simulación desde el marco de inteligibilidad de la cognición situada. Este objetivo se abordará teniendo presentes algunos elementos que son premisas dentro de la cognición situada, y que a manera de niveles de análisis permitirán abordar dicho objetivo. 
2.2.2.1. El software de simulación como entorno de realidad en la aprehensión de un contenido significativo

En la cognición situada el contenido y su significado son negociados, no impuestos, aquí toma relevancia su uso, más que la retención como elemento que posibilita la interacción con la situación. El contenido es una herramienta que se genera en dependencia de las necesidades situacionales que se desarrollan en la especificidad de la actividad. Por tanto, el contenido relevante dentro del desarrollo de una actividad es inherente a las necesidades emergentes en dicha actividad, y no sugerencias descontextualizadas.

El contenido junto con otros elementos, es la herramienta que le permite a los individuos construir el significado, porque el significado esta anclado en el contexto situacional, éste cobra su valor en situación. El contacto con la realidad es esencial en la selección y búsqueda de los contenidos, porque los contenidos cobran significado en su contexto real. Así mismo, las relaciones causales, los atributos sociales, los aspectos lingüísticos, entre otros, son categorizaciones que pertenecen a un contexto situacional.

En las actividades académicas, los entornos de simulación reemplazan en gran medida el contexto de realidad, generando un entorno propicio para el flujo de formas culturales elaboradas socialmente. La interpretación emergente, sólo tendrán significado desde la interacción con dicho entorno de realidad simulada; esto es, elementos del contexto que toman significado en la situación y que existen más allá de la simple memorización (Lacasa, 1994).

Como condición, se hace necesario que los entornos de simulación computarizada presenten una interfaz que posibilite al aprendiz la representación de un evento con las características que posibilitaría un sistema real.

Cuando un aprendiz se enfrenta a un evento, ya sea simulado o real, se haya en la necesidad de interpretar dicho evento. Esta primera interacción con una situación desconocida, obliga al aprendiz a interesarse por comprender los elementos de dicha problemática. En este momento se torna necesario para el aprendiz, incorporar un contenido que le posibilite encontrar significado al evento desconocido, lo que genera una actividad mental que se torna específica para la situación. 
Ahora bien, lo que configura que un entorno sea pertinente en la construcción y adecuación de los contenidos, es la especificidad situacional, cultural y de actividad; estos elementos pueden ser conseguidos mediante un adecuado diseño instruccional y un oportuno uso de la simulación computarizada. Si los contenidos son producto de la actividad, si el evento real proporciona el significado a los contenidos y si la interacción social permite la interpretación de dichos contenidos, es posible notar en los entornos de simulación computarizados de realidad, un excelente recurso para generar eventos específicos de interacción social y situacional.

En esta medida, los programas de simulación vistos como herramientas para instrumentar los métodos, son entornos que permiten a los aprendices la interacción con una realidad que les proporciona los eventos adecuados para encontrar sentido a lo que se debe incorporar como contenido significativo (herramientas de interacción con el contexto).

\subsubsection{La simulación, contextos de realidad en la generación de actividad cognitiva}

Antes, se hace necesario esclarecer el término contexto, ya que éste puede suponer lugar o aspectos físicos de un determinado espacio. "Un contexto se delimita por lo que la gente hace, donde y cuando lo hace $y$, en este sentido las personas que interactúan llegan a construir el contexto para los demás" (Lacasa, 1995). "Los contextos situacionales de actividad no se hallan determinados por el contexto físico. Es más, son creados por participantes en el contexto" (Wertsch, 1988, p. 223).

Al hacer alusión a este término, se hace referencia al contexto de actividad que integra los demás contextos; es decir, en el contexto de actividad está presente el contexto de representaciones culturales, sociales y situacionales. Así, el contexto de actividad es el entorno que se genera durante el desarrollo de la interacción del individuo con las condiciones que posibilitan o dificultan dicha actividad.

En la cognición situada, el contexto aporta ingredientes que permiten la construcción de significados, la especialización y comprobación del conocimiento, "la combinación de objetivos, herramientas y marco contextual (...), los cuales constituyen simultáneamente el contexto del comportamiento y las maneras en que puede decirse que la cognición está distribuida en ese contexto" (Salomon, 1993, p. 28). 
Al respecto, los entornos de simulación posibilitan un contexto de realidad que invita a los aprendices a generar actividad con relación a las condiciones simuladas que dentro de una actividad metodológica se desarrolla. En tal sentido, el entorno de simulación debe responder a las condiciones planeadas en la instrucción, a su vez, dicho entorno debe permitir al docente una amplia diversidad de secuencias y rutinas que permitan generar actividad con relación a los objetivos planeados. Por ejemplo, si se desea hacer énfasis en la construcción de procedimientos, el simulador debe facultar la repetición de rutinas y secuencias como método de dicha construcción.

Por otra parte, los entornos de simulación son el eje de conexión entre el conocimiento y el mundo, porque la actividad de los individuos se genera en la interacción con el mundo y las relaciones causales de esta interacción. En este caso, la simulación representa las estructuras de la realidad, desde la cual el aprendiz construye, ajusta y libera actividad con relación a las condiciones emergentes.

La perspectiva del aprendizaje situado, establece que para considerar, analizar o interpretar cualquier fenómeno de la práctica y/o cognitivo es imprescindible considerarlo como una actividad interactiva y en situación (Gremo \& Moore, 1993). Se centra, por tanto, en las estructuras de la realidad, en cómo guían y se condicionan mutuamente el comportamiento y la cognición evitando la diglosia entre conocimiento y mundo, en la actividad de los agentes, de los grupos y de las máquinas en situación y en contexto, en ajuste y la acomodación de la mente a las situaciones, a las interacciones sociales. (Lozares, 2000, pp. 97-131)

La actividad al marco de los entornos de simulación computarizada, ha de verse como la interacción con los artefactos e instrumentos bajo las circunstancias sociales que los envuelven y no sólo como interacción entre sujetos sociales (...). Los recursos e instrumentos, sobre todo los objetos informáticos y automatizados, son también mediadores y actuantes entre el mundo y los agentes. (Lozares, 2000, pp. 97-131).

No se debe olvidar que los contextos físicos también modifican la acción de los actores en la actividad. Para este caso, los simuladores computarizados facultan un determinado tipo de posibilidades que pueden ser diseñadas y manipuladas a voluntad para 
permitir que los aprendices generen actividad controlada a fines particulares. Este aspecto se torna en un elemento positivo para este caso, pues posibilita que la actividad educativa sea premeditada y planeada para que el estudiante responda de una manera determinada en relación con la situación y las posibilidades que el contexto le presenta. Con esto no se expresa que el simulador deba ser un plan secuencial que lleve de la mano al aprendiz, sino más bien debe ser un entorno de oportunidades situacionales diseñadas para permitir el fin.

En este sentido, Schman, L., presenta un aporte que parte de la crítica a los planes y sistemas de instrucción basados en ciencia cognitiva, donde las experiencias y situaciones de otros eventos y sujetos, son incorporadas como rutinas de instrucción. Esta apreciación surge tras investigaciones realizadas a grupos de aprendices en el manejo de máquinas fotocopiadoras, donde las mismas proporcionan a los usuarios de ayudas y rutinas instruccionales para asistir en el desempeño de las tareas a los novatos. Tras la investigación, "llegó a la conclusión de que las personas inexpertas no actúan según planes (ni siquiera cuando habían leído las instrucciones), sino sobre la base de conjuntos de destrezas previamente incorporadas que habían ido configurando en una trayectoria histórica de acciones similares" (Streibel, 1989, pp. 215-234). Estas acciones surgen en dependencia de la situación que se genera de la interacción con las maquinas y en directa relación con el evento particular. Tras su experiencia, Schuman llegó a afirmar que el aprendizaje es producto de la interacción con la situación, así mismo, cuestiona la posibilidad de aprendizaje significativo desde la interacción basada en planes y rutinas preestablecidas.

Streibel, M., tras referirse a la anterior investigación realizada por Schman, concluye: "nuestra experiencia fenomenológica nos dice que nuestro conocimiento supone una experiencia específica y contextual y que nuestras acciones se producen no desde planes construidos racionalmente, sino sobre la base de nuestras propias destrezas incorporadas, que son sensibles al contexto" (Streibel, 1989, pp. 215-234).

\section{Metodología}

\subsection{Diseño experimental}

Es posible estimar que existen numerosas experiencias que resaltan los efectos positivos sobre el uso de los simuladores en las actividades pedagógicas, sin embargo, se ha podido apreciar que los estudios existentes evalúan el potencial pedagógico de los entornos de simulación desde aspectos comunes en casi todas las investigaciones: 
- En la mayoría de experiencias se han evaluado las propiedades pedagógicas de los simuladores computarizados, versus las potencialidades pedagógicas de los entornos tradicionales de aprendizaje. Estos estudios arrojan resultados que resaltan las virtualidades de los entornos de simulación, en vista a la mejor comprensión de la complejidad dinámica de los sistemas que se estudian en las clases (Wolfe, 1975; Orlansky, \& String, 1977; Akpan, 2001; Pozo; Álvarez; Roble, \& Ascuy, 1998).

- Por otra parte, se ha evaluado el potencial de las simulaciones computarizadas con relación a su utilización dentro del proceso metodológico antes y después de la interacción con eventos reales; arrojando mejores resultados para la utilización de las simulaciones previas a las actividades reales (Domínguez, 1986).

- En otros estudios se ha comprobado que el uso de los simuladores computacionales reduce el tiempo necesario que requieren los aprendices para incorporar el conocimiento (Kinzie, Strauss \& Foss, 1993).

- En estudios donde se compara la simulación interactiva con la simulación de videocinta, se han detectado significativos resultados a favor de los grupos que usaron la simulación interactiva (Akpan, 2001).

- Un variado grupo de investigaciones demuestra que las actividades de laboratorio en el marco de la educación tradicional, pueden ser tan significativas como las actividades que desde la misma metodología se apoyan en contextos de simulación computarizada (Agnew \& Glen 1990; Chong, 1993; Rosado \& Herrero, 2006).

- Los estudios que se han desarrollado bajo cognición situada, con la ayuda de simulaciones como instrumento del método en la contextualización del aprendizaje, demuestran que las simulaciones proporcionan un contexto más significativo que el proporcionado por las actividades de clase tradicional (Learning Technology Center. 1992; Kilman, 1993; Bottge \& Hasselbring, 1998).

- En el mismo sentido, varias experimentaciones han demostrado que las actividades pedagógicas basadas en una metodología de la cognición situada, generan mejores resultados respecto a otras metodologías que se basan en el enfoque tradicional (Shao, 1999; Milrad, 2002; Granada, 1999).

Desde lo anterior, se aprecia que no existen muchas investigaciones que comparan los efectos que producen los entornos de simulación computarizada, frente a los efectos que generan los espacios de realidad, como instrumentos del método en la enseñanza y el aprendizaje de la física o de otras asignaturas. 
Al respecto, se considera pertinente verificar si estos entornos de realidad simulada por ordenador, pueden en algún momento ser tan significativos para la construcción del conocimiento, como los entornos de realidad que se generan dentro de un laboratorio verdadero.

Desde lo anterior, surge el siguiente cuestionamiento: La instrucción basada en una metodología del conocimiento situado dentro de una realidad simulada ¿puede ser tan significativa para la construcción del conocimiento, como la instrucción que desde la misma metodología se sitúa en contextos de laboratorio reales?

\subsection{Objetivos}

Buscando materializar los intereses particulares que genera la presente investigación, se hace necesario determinar los campos del conocimiento que serán evaluados dentro de la temática seleccionada- Ley de Ohm-. Por tanto, los siguientes son los objetivos que se establecen teniendo presente la construcción del conocimiento en el ámbito de conceptos, procedimientos y actitudes.

- Comparar y evaluar la eficacia de la simulación computarizada frente a la eficacia de las actividades desarrolladas con material real, como instrumentos del método en la construcción y aplicación del conocimiento conceptual y procedimental, con relación al aprendizaje de la física en estudiantes de secundaria.

- Comparar y evaluar la simulación computarizada frente a las actividades desarrolladas con material real, como instrumentos del método y como elementos que influyen en la actitud de los educandos frente al aprendizaje de la física.

- Comparar y Evaluar los resultados obtenidos por los aprendices en términos de descontextualización (transferencia) del aprendizaje, producto de una instrucción anclada en entornos de simulación, frente a los resultados obtenidos producto de la misma instrucción, anclada en entornos de realidad.

- Comparar y evaluar los resultados en cuanto a retención del aprendizaje conceptual y la actitud de los aprendices, pasados dos meses de concluida la instrucción.

Ahora bien, siguiendo el curso de la investigación, a continuación se presentan las hipótesis de partida. 


\subsection{Hipótesis del estudio}

Con el ánimo de establecer las hipótesis que han de ser contrastadas al final de la presente investigación, se considera pertinente hacer hincapié en las dos variables que condicionan su redacción. Por tanto, se tiene como variable independiente, la metodología empleada en el proceso instructivo y, como variable dependiente, el aprendizaje producto de las actividades propuestas. Este cometido será posible si se plantea comparar una situación experimental con una situación testigo.

\section{Cuadro 1. Relación de las hipótesis de partida.}

\begin{tabular}{|c|c|}
\hline Hipótesis 1: & $\begin{array}{l}\text { Los estudiantes que reciben instrucción basada en cognición situada, usando el } \\
\text { entorno de simulación computarizado como sustituto de la realidad, presentan el } \\
\text { mismo nivel de resultados en cuanto a construcción y reconstrucción del } \\
\text { aprendizaje conceptual, frente a los estudiantes que reciben la misma } \\
\text { metodología usando el laboratorio y material real como sustituto de la realidad. }\end{array}$ \\
\hline Hipótesis 2: & $\begin{array}{l}\text { Los estudiantes que reciben instrucción basada en cognición situada, usando el } \\
\text { entorno de simulación computarizado como sustituto de la realidad, presentan el } \\
\text { mismo nivel de resultados en cuanto a construcción y reconstrucción del } \\
\text { conocimiento en el ámbito de procedimientos, frente a los estudiantes que reciben } \\
\text { la misma metodología usando el laboratorio y material real como sustituto de la } \\
\text { realidad. }\end{array}$ \\
\hline Hipótesis 3: & $\begin{array}{l}\text { Los estudiantes que reciben instrucción desde una metodología de la } \\
\text { cognición situada apoyados con entornos de simulación, presentan igual } \\
\text { nivel en cuanto a la actitud de aprendizaje y al clima relacional y } \\
\text { motivacional, con relación a los estudiantes que reciben instrucción desde } \\
\text { la misma metodología apoyada con material real. }\end{array}$ \\
\hline Hipótesis 4: & $\begin{array}{l}\text { Los estudiantes que usan la simulación computarizada como instrumento del } \\
\text { método en la construcción del conocimiento, presentan resultados en cuanto a } \\
\text { descontextualización del aprendizaje, tan favorables como aquellos alumnos que } \\
\text { desarrollan actividades metodológicas con material real. }\end{array}$ \\
\hline Hipótesis 5: & $\begin{array}{l}\text { Después de pasados dos meses de recibida la instrucción, los alumnos que } \\
\text { usaron la simulación como instrumento del método pedagógico en la } \\
\text { construcción del conocimiento, presentan los mismos resultados en cuanto } \\
\text { a retención del aprendizaje conceptual, como el presentado por los alumnos } \\
\text { del grupo que empleó el método usando material real. }\end{array}$ \\
\hline Hipótesis 6: & $\begin{array}{l}\text { Después de pasados dos meses de recibida la instrucción, los alumnos que } \\
\text { usaron la simulación como instrumento del método en la construcción del } \\
\text { conocimiento, presentan resultados decrecientes respecto al clima } \\
\text { relacional y actitudinal, en la misma proporción que los estudiantes que } \\
\text { recibieron instrucción usando material real. }\end{array}$ \\
\hline
\end{tabular}

\subsection{Tipo de investigación}

Para abordar esta fase experimental, se han tenido presentes los lineamientos del diseño investigativo cuasiexperimental y se ha elegido un modelo con prueba-posprueba, 
grupos intactos. Este diseño se caracteriza por presentar dos grupos, uno experimental y otro control. Su esquema representativo es el siguiente:

Cuadro 2. Diseño cuasiexperimental con preprueba y posprueba.

\begin{tabular}{|l|c|c|c|}
\hline \multicolumn{1}{|c|}{ Grupos } & $\begin{array}{c}\text { Medida de la } \\
\text { pre-prueba }\end{array}$ & Tratamiento & $\begin{array}{c}\text { Medida de la } \\
\text { pos-prueba }\end{array}$ \\
\hline Experimental & $0_{1}$ & $\mathrm{X}$ & $0_{2}$ \\
\hline Control & $0_{1}$ & - & $0_{2}$ \\
\hline
\end{tabular}

Según la naturaleza de los datos, la presente investigación se enmarca dentro de un enfoque dominante, donde predomina el modelo cuantitativo, aunque algunos datos requieren de un análisis cualitativo.

Como temática dentro de los contenidos en la enseñanza y aprendizaje de la física, se ha tenido presente que ésta, más que memorización, exija la lógica y la aplicación de un aprendizaje consciente, significativo y acorde con el contexto situacional. A demás, otro elemento que ha condicionado la selección de la temática, es el simulador, ya que se hace necesario que éste cumpla con las condiciones fundamentales para ser empleado en la instrucción. Teniendo presentes estos elementos, se ha seleccionado para la presente investigación, el comportamiento de la corriente eléctrica dentro de los circuitos eléctricos de corriente continua; esto es, la interpreta y la aplicación de la de Ohm.

Para la actividad empírica se ha seleccionado una muestra conformada por 50 estudiantes, de los cuales 32 integraban el grupo experimental y 18 el grupo control. El primero usó el simulador como instrumento para la contextualización de las actividades pedagógicas y el segundo, con la misma finalidad, usó los contextos de laboratorio con material real.

\subsection{Diseño y aplicación de la metodología didáctica}

Antes de diseñar las actividades metodológicas, se ha hecho necesario establecer un resumido análisis sobre las concepciones erróneas que generalmente traen consigo los aprendices a la instrucción. Lo anterior se establece como punto de partida y elemento que guiará la planeación metodológica. 
Para este cometido se han tomado los aportes de autores como Osborne, R. \& Freyberg, P. (1998). Tras investigaciones realizadas con aprendices en Nueva Zelanda, estos autores han permitido una clasificación de dichas tendencias en cuatro modelos explicativos que concuerdan con otras investigaciones desarrolladas en otros lugares (Periago \& Bohigas, 2005). El aporte de los autores consiste en presentar las preconcepciones herradas, con relación al comportamiento de la corriente eléctrica dentro de un circuito. Por razones de espacio, solo se presentará una de estas clasificaciones, las otras tres se nombrarán.

La primera clasificación que exponen los autores se relacionan con el flujo de corriente eléctrica necesaria para que se encienda una bombilla dentro de un circuito eléctrico:

A) Modelo unipolar: Los individuos que presentan esta preconcepción, consideran que es suficiente una única línea para que se encienda la bombilla, y que generalmente ésta sale del polo positivo.

B) El modelo de choque de corriente: En este modelo, la corriente fluye desde los terminales de la pila en direcciones inversas, para luego encontrarse en el componente, donde chocan y se produce la corriente eléctrica.

C) Modelo de corriente atenuada: Este modelo está basado en otros conceptos mucho más evolucionados. La mayoría de estudiantes que razonan de esta forma, ya poseen conceptos básicos del funcionamiento y el comportamiento de la corriente en un circuito serie. Este preconcepto se basa en la creencia que la corriente, luego de pasar por el componente es consumida en parte y sale con menos fuerza. Por esta razón, explican el hecho que una bombilla encienda menos que otra o tengan menos brillo cuando se colocan una luego de la otra (serie). Cabe resaltar que este fenómeno se produce porque a mayor cantidad de bombillas, mayor resistencia al paso de corriente.

Así mismo, como estas preconcepciones se han tenido presentes otras tres clasificaciones que han condicionado la planeación de las actividades didácticas: II. La intensidad de corriente se debilita a medida que circula por un circuito, III. Relación entre voltaje y corriente, IV. Relación entre intensidad de corriente, con la diferencia de potencial y resistencia (Driver, Squires, Rushworth, \& Wood-Robinson, 1994); (Periago \& Bohigas, 2005). 


\subsection{Objetivos o propósitos instruccinales:}

- Establecer en los educandos elementos teóricos que les permitan comprender el funcionamiento y el comportamiento de los circuitos eléctricos.

- Posibilitar a los estudiantes la construcción significativa del aprendizaje conceptual y procedimental, necesario para la construcción de circuitos eléctricos seriales y paralelos con un cierto grado de dificultad.

- Calcular mediante la ley de Ohm el comportamiento y la relación existente entre la intensidad, la tensión y la resistencia dentro de circuitos series y paralelos.

- Transferir el aprendizaje adquirido a otro entorno diferente en el que éste fue aprendido.

\subsection{Planeación de las actividades}

La planeación de las actividades instruccionales se ha desarrollado bajo las premisas de la cognición situada, dentro de los objetivos anteriormente descritos.

Por otra parte y buscando hacer de la instrucción un evento situacional de interés para los estudiantes y acorde con su entorno social, se ha decidido usar como eje fundamental de la actividad, una situación problemática generada al interior del aula de clase. Esto, con base en la premisa que sustenta que toda actividad surge de la interacción consciente del individuo con las condiciones impuestas por el entorno. En palabras de Leóntiev, A. (1984), el objeto de la actividad es un verdadero motivo. Se sobreentiende que éste puede ser tanto material como ideal, tanto dado en la percepción como existente sólo en la imaginación, en el pensamiento. Lo fundamental es que detrás de cada motivo está siempre la necesidad. (p. 126)

Durante la fase de instrucción los estudiantes del grupo control trabajaron con material real, y los estudiantes del grupo experimental, trabajaron con el software para construcción de circuitos eléctricos, denominado Crocodile Clips 3. Al respecto, se garantizó que los elementos que posibilita el software fueran los mismos que posibilita el entorno real.

En las actividades, los estudiantes deberán resolver situaciones que emergen del contexto, donde la construcción de circuitos seriales y paralelos y la aplicación de la ley de OHM son esenciales. 


\subsection{Control de variables y aplicación de las pruebas}

La investigación fue rigurosamente controlada, teniendo presentes en su mayoría las variables que pueden influir en los resultados, así:

\section{Cuadro 3. Listado de variables contenidas en el estudio.}

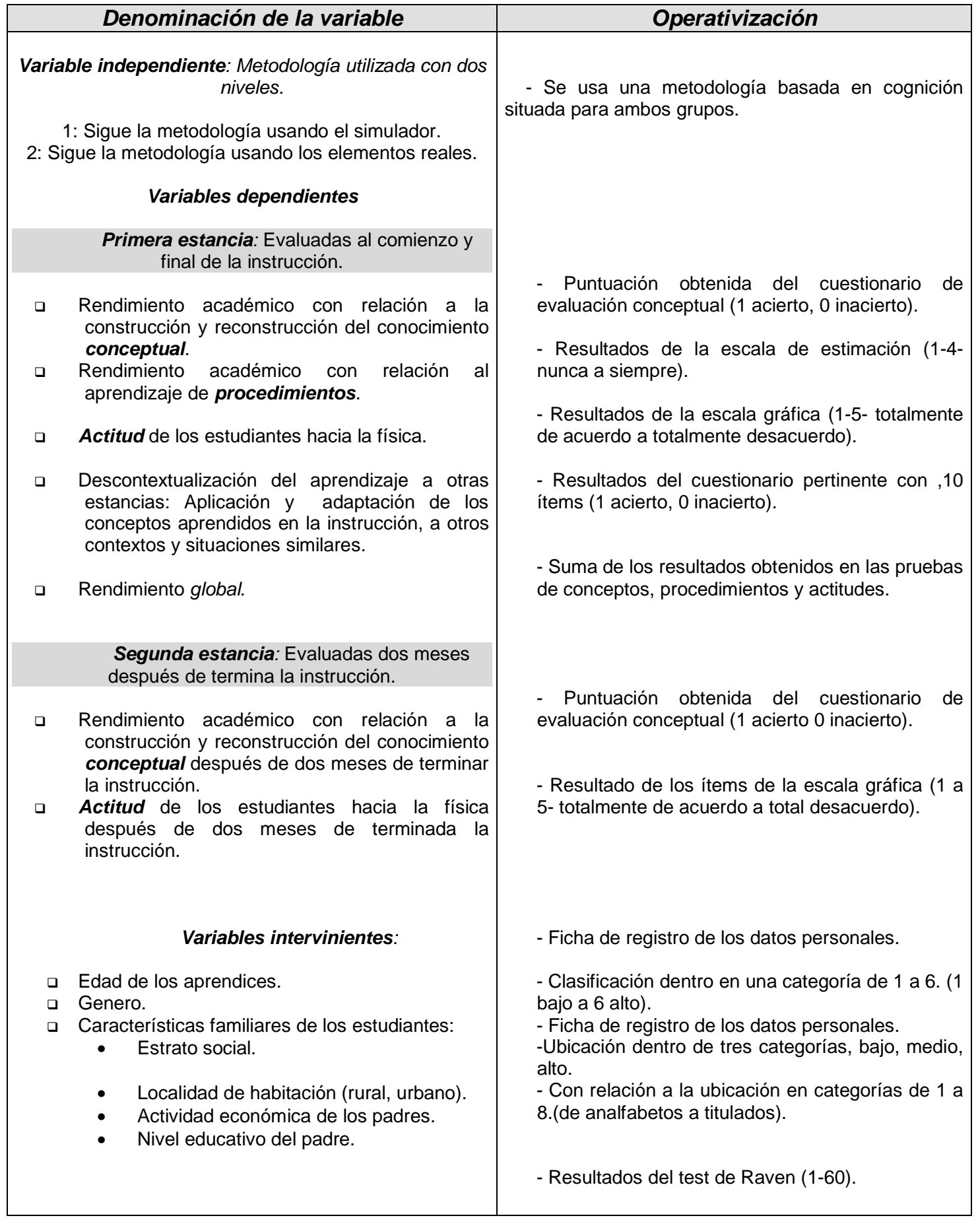




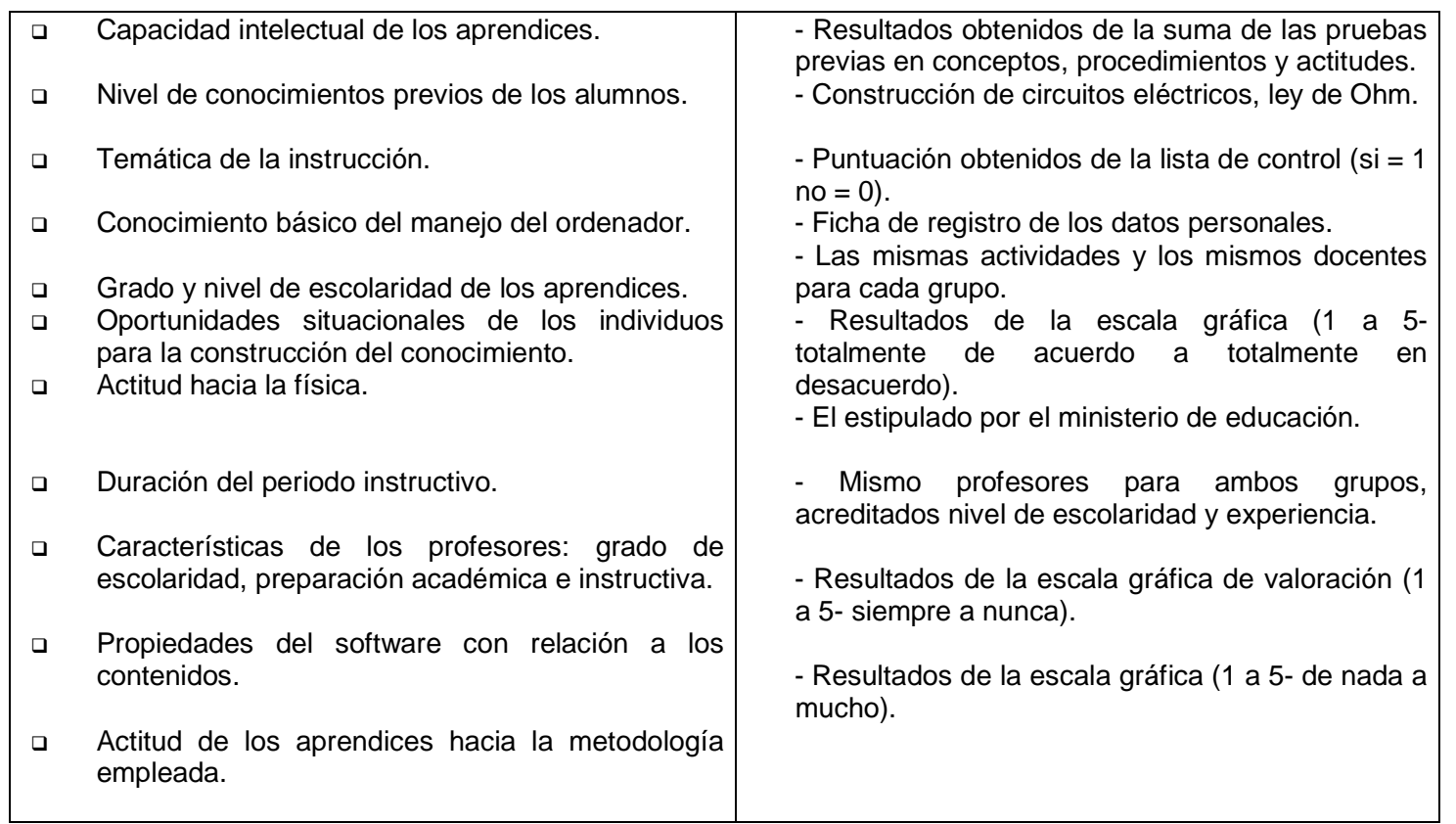

Para la aplicación de las pruebas se ha tenido presente de la siguiente secuencia:

Fase pretest o preinstrucción: En esta estancia se pretende medir el estado inicial de las variables dependientes, además, en esta fase se recopila información pertinente a comprobar la homogeneidad de los grupos entre sí.

Fase postest o postinstrucción: En esta estancia se recoge la información pertinente a determinar el aprendizaje adquirido como producto de la instrucción (conceptos, procedimientos y actitudes).

Fase retención del aprendizaje: En esta fase se recopila la información pertinente a constatar la existencia del aprendizaje, pasados dos meses de terminada la instrucción.

\subsection{Aplicación de las pruebas}

\subsubsection{La homogeneidad de los grupos, fase pretest}

En la primera fase, destinada a determinar la homogeneidad de los grupos, se ha podido observar que no hay diferencias significativas entre los grupos a un Nivel de Significación (NS) de 0,05 en las siguientes variables: 
Edad, Género, Características Familiares (nivel socioeconómico, actividad económica, nivel educativo del padre), Inteligencia General (factor $\mathrm{G}$ ).

\subsubsection{Resultado y análisis de las variables dependientes}

a) La homogeneidad con relación al nivel de conocimientos previos de los aprendices, conceptos y estructuras conceptuales, fase preinstrucción

El siguiente análisis surge desde la necesidad de verificar la homogeneidad en los grupos y establecer un momento inicial para las variables dependientes.

\section{Cuadro 4. Resultados del $t$ y la descriptiva para la puntuación total en la variable conocimientos previos con relación al conocimiento conceptual.}

\begin{tabular}{|l|c|c|c|c|c|}
\hline \multicolumn{1}{|c|}{ Grupo } & N & Media & $\begin{array}{c}\text { Desviación } \\
\text { típica. }\end{array}$ & $\boldsymbol{t}$ & $\boldsymbol{p}$ \\
\hline Experimental & 32 & 7,656 & 3,268 & 2,174 & 0,035 \\
\hline Control & 18 & 5,666 & 2,786 & & \\
\hline
\end{tabular}

Luego de hacer un análisis por ítem, se hace un análisis para toda la prueba. Los resultados de la prueba $t$, corroboran lo observado en el análisis por ítems, los grupos no son homogéneos con relación a la variable conceptos y estructuras conceptuales. Al respecto, se hace necesario realizar un análisis de covarianza (ANCOVA), con el fin de contrarrestar su efecto en la fase postest sobre la variable posconceptos, el cual se muestra más adelante.

b) Evaluación del conocimiento existente con relación a los procedimientos, fase preinstrucción

Cuadro 5. Resultados del $t$ y la descriptiva para la variable conocimientos previos con relación a los procedimientos, total de la prueba.

\begin{tabular}{|l|c|c|c|c|c|}
\hline \multicolumn{1}{|c|}{ Grupo } & $\mathbf{N}$ & Media & $\begin{array}{c}\text { Desviación } \\
\text { típica. }\end{array}$ & $\boldsymbol{t}$ & $\boldsymbol{p}$ \\
\hline Experimental & 32 & 26,281 & 1,800 & 1,122 & 0,903 \\
\hline Control & 18 & 26,222 & 1,308 & & \\
\hline
\end{tabular}

Los resultados del $t$ para el total de la prueba, demuestran que la hipótesis nula se acepta, por tanto, se puede afirmar que los grupos son homogéneos con relación al conocimiento previo en el ámbito de procedimientos. 
c) Evaluación de la actitud previa de los alumnos con relación a la asignatura de física

\section{Cuadro 6. Resultados para el total de la prueba de conocimientos previos con relación} a la actitud de los estudiantes hacia la física, fase preinstrucción.

\begin{tabular}{|l|c|c|c|c|c|}
\hline \multicolumn{1}{|c|}{ Grupo } & $\mathbf{N}$ & Media & $\begin{array}{c}\text { Desviación } \\
\text { típica. }\end{array}$ & $\boldsymbol{t}$ & $\boldsymbol{p}$ \\
\hline Experimental & 32 & 80,718 & 14,644 & 0,549 & 0,586 \\
\hline Control & 18 & 83,166 & 16,008 & & \\
\hline
\end{tabular}

Los resultados de la prueba demuestran que no hay diferencias significativas entre los grupos a un Nivel de Significación (NS.) de 0,05. Por esto, se puede afirmar que los grupos son homogéneos con relación a la variable actitud previa hacia la física.

Hasta el momento se ha podido comprobar que los grupos son homogéneos con relación a las variables previamente definidas, excepto con la variable conocimientos previos en cuanto a conceptos y estructuras conceptuales. Al respecto, como ya se ha mencionado, es pertinente controlar los efectos de la variable conceptos en la fase pretest, sobre los resultados para esta misma variable en la fase postest. Este análisis se muestra más adelante al presentar los resultados postest.

3.9.2. Análisis comparativo entre grupos, con el fin de establecer el cambio de las variables dependientes en la fase postinstrucción

a) Comparación entre grupos sobre el rendimiento académico con relación a la construcción y reconstrucción del conocimiento conceptual, una vez finalizada la instrucción

\section{Cuadro 7. Resultados del t para el total de la prueba con relación a la variable conceptos postinstrucción}

\begin{tabular}{|l|c|c|c|c|c|}
\hline \multicolumn{1}{|c|}{ Grupo } & N & Media & $\begin{array}{c}\text { Desviación } \\
\text { típica. }\end{array}$ & $\boldsymbol{t}$ & $\boldsymbol{p}$ \\
\hline Experimental & 32 & 14,44 & 3,501 & 1.697 & 0,096 \\
\hline Control & 18 & 12,83 & 2,595 & & \\
\hline
\end{tabular}

Aunque se presentaron diferencias significativas entre algunos de los ítems, en los resultados obtenidos para el total de la prueba no se observan diferencias significativas para la variable conceptos postinstrucción (posconceptos). 
Cabe recordar que en la prueba de homogeneidad (pretest), la comparación de los grupos con relación a esta variable presenta diferencias significativas a favor del grupo experimental. Por tanto, se hace necesario controlar el efecto de dicha deferencia en el resultado del postest. Por lo que se torna pertinente realizar un control para esta variable, a través de una prueba estadística denominada Análisis de Covarianza (ANCOVA):

\section{Cuadro 8. Análisis de covarianza para la variable conceptos} (Pruebas de los efectos inter-sujetos).

\begin{tabular}{|r|c|c|c|c|c|}
\hline Variable dependiente: posconceptos total. \\
\hline Fuente & $\begin{array}{c}\text { Suma de } \\
\text { cuadrados } \\
\text { tipo I }\end{array}$ & $\boldsymbol{G I}$ & $\begin{array}{c}\text { Media } \\
\text { cuadrátic } \\
\boldsymbol{a}\end{array}$ & $\boldsymbol{F}$ & $\boldsymbol{- p}$ \\
\hline $\begin{array}{r}\text { Modelo } \\
\text { corregido }\end{array}$ & $114,119(\mathrm{a})$ & 2 & 57,059 & 6,543 &, 003 \\
\hline Intersección & 9604,980 & 1 & 9604,980 & 1101,324 &, 000 \\
\hline $\begin{array}{r}\text { Preconceptos } \\
\text { total }\end{array}$ & 108,148 & 1 & 108,148 & 12,400 &, 001 \\
\hline Grupo & 5,971 & 1 & 5,971 & 0,685 & 0,412 \\
\hline Error & 409,901 & 47 & 8,721 & & \\
\hline Total & 10129,000 & 50 & & & \\
\hline Total corregida & 524,020 & 49 & & & \\
\hline a R cuadrado $=0,218(\mathrm{R}$ cuadrado corregida $=0,184)$. & & \\
\hline
\end{tabular}

Los resultados del análisis de covarianza demuestran que: Con relación al valor de $p$, no hay diferencias significativas para la variable posconceptos a un NS de 0,05 , luego de quitar el efecto de la variable preconceptos. Así, la Ho (no hay diferencias significativas entre los grupos con relación a la variable posconceptos), no se rechaza. Por lo que se puede afirmar que, al comparar los grupos con relación al aprendizaje conceptual en la fase postest, no se aprecian diferencias significativas a un NS de 0,05.

b) Comparación entre grupos sobre el rendimiento académico con relación a la construcción del conocimiento procedimental, una vez finalizada la instrucción

Cuadro 9. Resultados del t para la variable procedimientos, total de la prueba, fase post instrucción.

\begin{tabular}{|l|c|c|c|c|c|}
\hline $\begin{array}{c}\text { Prueba total } \\
\text { Grupo }\end{array}$ & N & Media & $\begin{array}{c}\text { Desviación } \\
\text { típica. }\end{array}$ & $\boldsymbol{t}$ & $\boldsymbol{p}$ \\
\hline Experimental & 32 & 66,75 & 11,542 & 0,669 & 0,507 \\
\hline Control & 18 & 64,39 & 12,729 & & \\
\hline
\end{tabular}


En los resultados se aprecia que no existen diferencias significativas para la variable procedimientos, porque a pesar de que en tres de sus ítems se presentaron diferencias significativas, este hecho no se ha visto reflejado en el total de la prueba.

c) Comparación entre grupos sobre el cambio de actitud inicial con relación a la fase postinstrucción

\section{Cuadro 10. Prueba $t$ de las muestras independientes para la variable actitud hacia la física, total de la prueba.}

\begin{tabular}{|c|c|c|c|c|c|c|}
\hline Grupo & Media & $\boldsymbol{N}$ & $\begin{array}{c}\text { Desviación } \\
\text { típ. }\end{array}$ & $\begin{array}{c}\text { Error típ. } \\
\text { de la media }\end{array}$ & $\boldsymbol{t}$ & $\boldsymbol{p}$ \\
\cline { 1 - 7 } Experimental & 83.88 & 32 & 9.401 & 1.662 & \multirow{2}{*}{$-0,805$} & 0,425 \\
\cline { 2 - 7 } Control & 86.28 & 18 & 11.345 & 2.676 & \\
\hline
\end{tabular}

Los resultados presentan una ligera ventaja del grupo control frente al grupo experimental, esto se puede divisar en los valores de la media. Sin embargo, según el valor de p, la diferencia no resulta ser significativa. Por consiguiente, se puede afirmar que no hay diferencias significativas entre los grupos con relación al cambio actitudinal visto como producto de la instrucción.

d) Resultados de la transferencia del aprendizaje a otros contextos similares.

Cuadro 11. Resultados de t para el total de la prueba correspondiente a la descontextualización del aprendizaje.

\begin{tabular}{|l|l|c|c|c|c|}
\hline \multicolumn{1}{|c|}{ Grupo } & $\mathbf{N}$ & Media & $\begin{array}{c}\text { Desviación } \\
\text { típica. }\end{array}$ & $\boldsymbol{t}$ & $\boldsymbol{P}$ \\
\hline Experimental & 32 & 5,25 & 2,064 & 1,325 & 0,192 \\
\cline { 1 - 4 } Control & 18 & 4,44 & 2,064 & & \\
\hline
\end{tabular}

Como se observa en el cuadro, no existen diferencias significativas para la variable transferencias del aprendizaje.

3.9.3. Análisis de los resultados con relación a la retención del aprendizaje, pasados dos meses de concluida la instrucción (conceptos y actitudes).

a) Comparación de la retención del aprendizaje conceptual pasados dos meses de concluida la instrucción, grupos experimental y control 
Cuadro 12. Resultados de la comparación de grupos en la prueba de conceptos, dos meses de concluida la instrucción, total de la prueba.

\begin{tabular}{|l|c|c|c|c|c|}
\hline Total prueba & $\mathbf{N}$ & Media & $\begin{array}{c}\text { Desviación } \\
\text { típica. }\end{array}$ & $\boldsymbol{t}$ & $\boldsymbol{p}$ \\
\hline Experimental & 32 & 14,29 & 3,778 & 2,569 & 0,013 \\
\hline Control & 18 & 11,33 & 3,955 & & \\
\hline
\end{tabular}

La gráfica muestra de una forma más clara la diferencia encontrada.

Gráfico 1. Resultados de la comparación de grupos en la prueba de conceptos, dos meses de concluida la instrucción, total de la prueba.

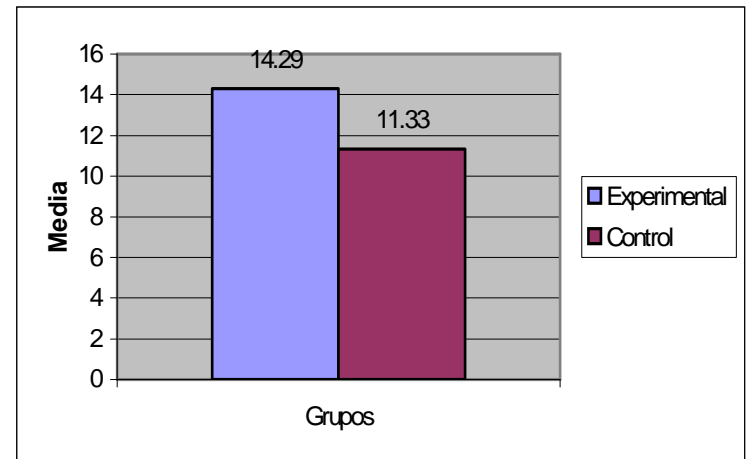

Al hacer una comparación entre los grupos teniendo presente el puntaje total de la prueba, se presentan diferencias significativas a favor del grupo experimental.

Al respecto, se puede afirmar que después de dos meses de concluida la instrucción, los estudiantes del grupo experimental presentan mejores resultados con relación a la retención del aprendizaje en conceptos frente al grupo control.

b) Cambio actitudinal pasados dos meses de concluida la instrucción, grupos experimental y control

Cuadro 13. Resultados de t para el total de la prueba correspondiente a la retención de la actitud pasados dos meses de terminada la instrucción.

\begin{tabular}{|l|c|c|c|c|c|}
\hline \multicolumn{1}{|c|}{ Grupo } & $\mathbf{N}$ & Media & $\begin{array}{c}\text { Desviación } \\
\text { típica. }\end{array}$ & $\boldsymbol{t}$ & $\boldsymbol{p}$ \\
\cline { 1 - 4 } Experimental & 32 & 79,13 & 16,238 & 0,441 & 0,661 \\
\hline Control & 18 & 77,22 & 11,117 & & \\
\hline
\end{tabular}


En los resultados, se corrobora lo observado en el análisis por ítems, no hay diferencias significativas entre los grupos con relación a la actitud que presentan los estudiantes hacia la física, pasados dos meses de concluida la instrucción.

\section{Contraste de hipótesis}

A continuación se presenta el contraste de las hipotes planteadas al comienzo del presente trabajo (hipótesis de partida), con los resultados obtenidos del análisis estadístico pertinente.

\section{Cuadro 14. Contraste de las hipótesis de la investigación con los resultados obtenidos.}

\begin{tabular}{|c|c|c|}
\hline Objetivo & Hipótesis de partida & Resultados y conclusiones \\
\hline & $\begin{array}{l}\text { Hipótesis 2: Los estudiantes que } \\
\text { reciben instrucción basada en cognición } \\
\text { situada, usando el entorno de } \\
\text { simulación computarizado como } \\
\text { sustituto de la realidad, presentan el } \\
\text { mismo nivel de resultados en cuanto a } \\
\text { construcción y reconstrucción del } \\
\text { conocimiento en el ámbito de } \\
\text { procedimientos, frente a los estudiantes } \\
\text { que reciben la misma metodología } \\
\text { usando el laboratorio y material real } \\
\text { como sustituto de la realidad. }\end{array}$ & $\begin{array}{l}\text { Para la variable procedimientos, los } \\
\text { resultados no presentan diferencias } \\
\text { significativas ( } \mathrm{t}=0,669 ; \mathrm{p}=0,507) \text {, al } \\
\mathrm{NS} \text { de } 0,05 \text {, por consiguiente, esta } \\
\text { hipótesis no se rechaza. }\end{array}$ \\
\hline
\end{tabular}




\begin{tabular}{|c|c|c|}
\hline $\begin{array}{l}\text { 3) Comparar y evaluar los resultados } \\
\text { obtenidos por los aprendices en } \\
\text { términos de descontextualización del } \\
\text { aprendizaje, producto de una } \\
\text { instrucción anclada en entornos de } \\
\text { simulación, frente a los resultados } \\
\text { obtenidos producto de la misma } \\
\text { instrucción, anclada en entornos de } \\
\text { realidad. }\end{array}$ & $\begin{array}{l}\text { Hipótesis 4: Los alumnos que usan la } \\
\text { simulación computarizada como } \\
\text { instrumento del método en la } \\
\text { construcción del conocimiento, } \\
\text { presentan resultados en cuanto a } \\
\text { descontextualización del aprendizaje, } \\
\text { tan favorables como aquellos alumnos } \\
\text { que desarrollan actividades } \\
\text { metodológicas con material real. }\end{array}$ & $\begin{array}{l}\text { En esta variable las diferencias por } \\
\text { ítem se presentan a favor de ambos } \\
\text { grupos, dos ítems para el grupo } \\
\text { experimental y un ítem para el grupo } \\
\text { control. Sin embargo, finalmente no se } \\
\text { presentan diferencias significativas } \\
\text { para el total de la prueba. } \\
\text { Por tanto, la hipótesis cuatro no se } \\
\text { rechaza. }\end{array}$ \\
\hline \multirow[t]{2}{*}{$\begin{array}{l}\text { 4) Evaluar y comparar los resultados } \\
\text { en cuanto a retención del aprendizaje } \\
\text { conceptual y la actitud de los } \\
\text { aprendices, pasados dos meses de } \\
\text { concluida la instrucción. }\end{array}$} & $\begin{array}{l}\text { Hipótesis 5: Después de pasados dos } \\
\text { meses de recibida la instrucción, los } \\
\text { alumnos que usaron la simulación como } \\
\text { instrumento del método pedagógico en } \\
\text { la construcción del conocimiento, } \\
\text { presentan los mismos resultados en } \\
\text { cuanto a retención del aprendizaje } \\
\text { conceptual, como el presentado por los } \\
\text { alumnos del grupo que empleó el } \\
\text { método usando material real. }\end{array}$ & $\begin{array}{l}\text { Se han presentado diferencias } \\
\text { significativas para el análisis por ítem y } \\
\text { para el total de la prueba, diferencia } \\
\text { que está a favor del grupo experimental } \\
\text { Así, con un } t=2,569 \text { y } p=0,013 \text {, se } \\
\text { establece la diferencia a favor del } \\
\text { grupo experimental con una media de } \\
14,29 \text { sobre } 11,33 \text { para le grupo } \\
\text { control. } \\
\text { Desde lo anterior, la hipótesis cinco se } \\
\text { rechaza. }\end{array}$ \\
\hline & $\begin{array}{l}\text { Hipótesis 6: Después de pasados dos } \\
\text { meses de recibida la instrucción, los } \\
\text { alumnos que usaron la simulación como } \\
\text { instrumento del método en la } \\
\text { construcción del conocimiento, } \\
\text { presentan resultados decrecientes } \\
\text { respecto al clima relacional y actitudinal, } \\
\text { en la misma proporción que los } \\
\text { estudiantes que recibieron instrucción } \\
\text { usando material real. }\end{array}$ & $\begin{array}{l}\text { Cabe resaltar que al comparar los } \\
\text { resultados en el postest y dos meses } \\
\text { después de terminada la instrucción } \\
\text { para un mismo grupo, el grupo } \\
\text { experimental se mantiene igual, } \\
\text { mientras que el grupo control ha } \\
\text { disminuido significativamente. Esta } \\
\text { disminución del grupo control no ha } \\
\text { afectado los resultados en la } \\
\text { comparación entre grupos. así, para la } \\
\text { variable actitud hacia la física, no se } \\
\text { presentan diferencias significativas a } \\
\text { un NS de } 0,05 \text { ( } t=0,441 ; p=0,661) \text {. } p \\
\text { Por lo tanto, la hipótesis seis, no se } \\
\text { rechaza.. }\end{array}$ \\
\hline
\end{tabular}

Ahora bien, una vez presentados los resultados que son producto del análisis estadístico aplicado a los datos, es pertinente pasar al siguiente apartado. En él se presentan las conclusiones generales que se derivan de la presente investigación.

\section{Conclusiones}

Los resultados del análisis por ítem se distribuyen para ambos grupos, con una cierta inclinación hacia el grupo experimental. En dichos resultados se observa que los estudiantes del grupo experimental, han incorporado mejor los conceptos que se relacionan con la construcción de circuitos eléctricos seriales y la aplicación de la ley de Ohm, frente a los estudiantes del grupo control. Al respecto, cabe establecer una relación entre el uso de los simuladores y los resultados observados.

Antes, es necesario recordar que un concepto es un aprendizaje que se establece y se encaja perfectamente dentro de una estructura de significados que le posibilitan su 
interpretación. Su "grado de comprensión alcanzado, dependerá no sólo de la claridad y organización de los materiales presentados, (...) sino de su relación con los conocimientos previos activados en el aprendiz y la reflexión sobre esa relación conceptual generada en el aprendiz por la actividad' (Pozo, 1996, p. 270). El concepto se adquiere gradualmente y puede presentar diferentes niveles de interpretación, lo que hace que dicho concepto sea contexto dependiente.

Al respecto, el grupo experimental, al igual que el grupo control, tienen las mismas posibilidades para que se produzca un aprendizaje declarativo y posteriormente un aprendizaje conceptual. Sin embargo, el grupo experimental puede generar mayores eventos que posibilitan incrementar las oportunidades para generar relaciones causales entre el evento y su significado. El simulador permite a los aprendices del grupo experimental, la construcción de diversos modelos representativos de los circuitos eléctricos y así mismo la posibilidad de hacer comprobaciones, en mayor medida que el grupo control.

Respecto a la relación significativa que el concepto debe representar en la estructura del aprendiz, ambos grupos, desde la metodología aplicada y desde las posibilidades situacionales, en algún momento encuentran el significado dentro de la actividad.

Los resultados arrojados luego de aplicar los análisis estadísticos han permitido establecer las siguientes conclusiones:

1) La cognición situada permite el ancla de las actividades y posibilita la construcción consciente y significativa del conocimiento.

2) El simulador permite magnificar el aprendizaje conceptual, cuando el factor tiempo interviene en las relaciones causales que posibilitan su aprehensión.

3) En la construcción del aprendizaje de procedimientos, un laboratorio real puede ser reemplazado, al menos en algunos casos, por un entorno de simulación computarizada.

4) Los entornos de simulación pueden reemplazar los contextos de laboratorio real, en el momento que se desee mejorar la transferencia (descontextualización) del aprendizaje. 
5) El aprendizaje mediado por entornos de simulación, posibilita la retención del aprendizaje a mediano plazo en mayor proporción que el aprendizaje mediado por entornos de laboratorio real.

6) Las simulaciones computarizadas pueden generar contextos significativos de interacción y construcción consciente del conocimiento, de la misma forma que lo han posibilitado los contextos de laboratorio real.

\section{Referencias}

Agnew, David \& Shinn, Glen. (1990). Effects of Simulation on Cognitive Achievement in Agriculture Mechanics. Journal of Agricultural Education, 31 (2), 12-16.

Akpan, Joseph. (2000). Using a Computer Simulation Before Dissection to Help Students Learn Anatomy. Journal of Computers in Mathematics and Science Teaching, 19 (3): 297-313. Recuperado el 15 de noviembre de 2005 de http://www.aace.org/dl/files/jcmst/jcmst-19-03-297.pdf

. (2001). Issues Associated with Inserting Computer Simulations into Biology Instruction: A Review of the Literature. Electronic Journal of Science Education, 5 (3). Recuperado el 01 de enero de 2006 de http://unr.edu/homepage/crowther/ejse/akpan.html.

Amaya, Germán. (2007). Potencialidades Pedagógicas de los Entornos de Simulación, Desde la Perspectiva de la Cognición Situada. Salamanca: Ediciones Universidad de Salamanca.

Arias, Leandro. (2004). La Simulación Computarizada como Procedimiento Metodológico en la Enseñanza y el Aprendizaje de la Electrónica. Recuperado el 26 Marzo, 2005, de http://www.monografias.com/trabajos13/simucom/simucom.shtml?monosearch.

Ausubel, David. (1976). Psicología Educativa. Un Punto de Vista Cognitivo. México: Trillas.

Bettetini, Gianfranco \& Colombo, Fausto. (1995). Las Nuevas Tecnologías de la Comunicación ( $1^{\text {a } e d i c i o ́ n) . ~ B a r c e l o n a: ~ P a i d o ́ s . ~}$

Brown, John, Collins, Allan \& Duguid, Paul. (1989). Situated Cognition and the culture of learning. Educational Researcher, 18 (1), 33-42.

Bruner, Jerome. (1984). Acción, Pensamiento y Lenguaje. Madrid: Alianza Editores.

Caicedo, Surgey, Castillo, Olga, Fernández, Claudia. (2005). Influencia de la Tecnologías Educativa en el Proceso de Formación Integral de los Agentes Educativos Básicos. Revista Tecnologías de Avanzada, 1(5), 47-55.

Chaiklin, Seth \& Lave, Jean. (2001). Estudiar las Prácticas. Argentina: Amorrortu. 
Chong, Toh. (1993). Use of Microcomputer Simulations to Overcome Student Misconceptions About Displacement of Liquids. Conferencia anual. Australian Association for Research in Education. Recuperado el 10 de diciembre, 2005 de http://www.aare.edu.au/93pap/tohs93222.txt

Clancey, William. (1995). A Tutorial on Situated Learning. Proceedings of the International Conference on Computers and Education (Taiwan) Charlottesville, 49-70. Recuperado el 18 de Abril, 2004, de http://www.cogprints.ecs.soton.ac.uk/archive/00000323/00/139.htm

Coll Cesar, Palacios, Jesús, Marchesi, Álvaro. (1990). Desarrollo Psicológico y Educación II. Psicología de la Educación. Madrid: Alianza.

De Pablos, Pons \& Segura, Jiménez. (1998). Nuevas Tecnologías, Comunicación Audiovisual y Educación. Barcelona: Cedecs.

Delval, Juan. (1986). Niños y Máquinas. Los Ordenadores y la Educación. Madrid: Alianza.

Dewey, John. (1989). Cómo Pensamos: Nueva Exposición de la Relación entre Pensamiento Reflexivo y Proceso Educativo. Barcelona: Paidós.

Driver, Rosalind, Squires, Ann, Rushworth, Peter \& Wood-Robinson, Valerie. (1994). Dando Sentido a la Ciencia en Secundaria. Investigaciones Sobre las Ideas de los Niños. Madrid, Visor.

García Carrasco, Joaquín. (2004). La Compresión de la Vivienda Como un Dominio Vital de los Seres Humanos. Revista Española de Pedagogía. 228 (3), 229-256.

García Carrasco, Joaquín. (2007). Leer en la Cara y en el Mundo. Los dos Libros del Ser Humano. Barcelona: Herder.

García Del Dujo, Ángel \& Martín García, Antonio. (2002). Características Pedagógicas de los Entornos Virtuales de Aprendizaje. Teoría de la Educación. Revista Interuniversitaria, 14, 67-92.

Garrido, Antoni. (2003). El Aprendizaje como Identidad de Participación en la Práctica de una Comunidad Virtual. Recuperado el 18 de Febrero, 2004, de http://www.uoc.edu/in3/dt/20088/index.html.

González, Víctor. (1990). Teoría y Práctica de los Medios de Enseñanza. La Habana: Pueblo y Educación.

Hung, David. (2002). Situated Cognition and Problem-based Learnig: Implications for Learning and Instructions with Tecnology. Journal of Interactive Learning Research, 13 (4), 393-414.

Kilman, David. (1993). Technology and Situated Cognition. Tecnology \& Learnig, 14 (1), 86(1). 
Kinzie, Mable, Strauss, Richard, \& Foss, Michael. (1993). The Effects of an Interactive Dissection Simulation on the Performance and Achievement of High School Biology

Students. Journal of Research in Science Teaching, 30(8), 989-1000.

Lacasa, Pilar. (1994) Aprender en la Escuela, Aprender en la Calle. Madrid: Visor.

Lave, Jean \& Wenger, Etienne. (1991). Situated Learnig Legitimate Peripheral Participation. USA: Cambridge University Press.

Learning Technology Center. (1992). The Aventures of Jasper Woodbury. Recuperado el 20 de Junio, 2004, de http://peabody.vanderbilt.edu/projects/funded/jasper/Jasperhome.html.

Leontiev, Aleksei. (1974). The Problem of Activity in Psychology. Soviet Psychology 13 (2), 4-33.

(1984). Actividad Conciencia y Personalidad. México: Cartago.

Leontiev, Aleksei, Luria, Aleksandr. \& Vygotsky, Lev. (1973). Psicología y Pedagogía. Madrid: Akal.

Lozares, Carlos. (2000). La Actividad Situada y/o el Conocimiento Socialmente Distribuido. Recuperado el 28 de Agosto, 2003, de http://www.bib.uab.es/pub/papers/02102862n62p97pdf.

Martí, Eduardo. (1992). Cuadernos de Educación. Aprender con Ordenadores en la Escuela. Barcelona: Ice-Horsori.

Martínez, Silvio \& Requena, Alberto. (1986). Dinámica de Sistemas. Simulación por Ordenador. Madrid: Alianza.

Michelsen, Carlos. (2004). Eficacia de la Simulación. II Simposio Internacional e-learning: Soluciones aplicadas a la formación, capacitación y gestión del conocimiento. Recuperado el 10 de Octubre, 2005, de http://www.tecsup.edu.pe/webuds/web/simposios/elearning/principal/index.htm

Milrad, Marcelo. (2002). Using Construction Kits, Modeling Tools and System Dynamics Simulations to Support Collaborative Discovery Learning. Educational Technology \& Society, 5 (4), 2-15. Recuperado el 02 de Enero, 2006, de http://ifets.ieee.org/periodical/vol 4 2002/milrad.html.

Molina, Lourdes. (1997). Participar en Contextos de Aprendizaje y Desarrollo. Barcelona: Paidós.

Periago, María Cristina \& Bohigas, Xavier. (2005). Persistencia de las Ideas Previas Sobre Potencial Eléctrico, Intensidad de Corriente y Ley de Ohm en los Estudiantes de Segundo Curso de Ingeniería. Revista Electrónica de Investigación Educativa, 7 (2), Recuperado el 30 de Enero, 2005, de http://redie.uabc.mx/vol7no2/contenidoperiago.html. 
Pozo, Esperanza., Álvarez, Liduvina., Roble, Alina. \& Ascuy, Miguel. (1998). Las Simulaciones Computarizadas en la Enseñanza de Enfermería [Versión electrónica]. Revista Cubana de Enfermería, 14 (2), 112-116 Recuperado el 25 de febrero de 2006 en http://scielo.sld.cu/scielo.php?pid=S0864$\underline{03191998000200006 \& \text { script }=\text { sci abstract }}$

Pozo, Juan \& Gómez, Miguel. (1998). Aprender y Enseñar Ciencia. Del Conocimiento Cotidiano al Conocimiento Científico. Madrid: Morata.

(1996). Aprendices y Maestros. Madrid: Alianza.

Rogoff, Barbara. (1993). Aprendices del Pensamiento. Barcelona: Paidós.

Rosado, Luis. \& Herrero, Juan. (2006). Nuevas Aportaciones Didácticas de los Laboratorios Virtuales y Remotos en la Enseñanza de la Física. The 3rd International Conference on Multimedia and Information and Comunication Technologies in education. Recuperado el 19 de Octubre, 2006, de http://www.formatex.org/micte2005/286.pdf

Salomon, Gavriel. (1993). Cogniciones Distribuidas: Consideraciones Psicológicas y Educativas. Buenos Aires, Amorrortu.

Sancho, Joana. (1994). Para una Tecnología Educativa. Barcelona, Horsori.

Streibel, Michael. (1989). Diseño Instructivo y Aprendizaje Situado: ¿Es Posible un Maridaje? Revista de Educación, 289, 215-234.

Tarifa, Eric. (2005). Teoría de Modelos y Simulación. Introducción a la Simulación. Facultad de Ingeniería, Universidad Nacional de Jujuy. Recuperado el 05 de Mayo, 2005, de la http://www.modeladoeningenieria.edu.ar/unj/tms/apuntes/cp1.pdf

Vygotsky, Lev. (1979). El Desarrollo de los Procesos Psicológicos Superiores. Barcelona, Crítica. (1984). Obras Escogidas IV. Moscú, Visor.

Wenger, Etienne. (2001). Comunidades de Práctica. Barcelona, Paidós.

Wertsch, James. (1988). Vygotsky y la Formación Social de la Mente. Barcelona, Paidós. (1993). Voces de la Mente. Madrid, Visor.

\section{Agradecimientos:}

La realización de este trabajo de investigación no hubiese sido posible sin el aporte de:

-Dr. Álvaro González Joves, Rector de la Universidad de Pamplona Col.

-Doctores: Joaquín García Carrasco y María José Rodríguez Conde, Directores de la tesis Doctoral.

-Mg. Gloria del Rosario Galilea Ramírez. 